\title{
Graphical user interface based model for transmission line performance implementation in power system
}

\author{
Nur Ashida Salim¹, Hasmaini Mohamad², Zuhaila Mat Yasin ${ }^{3}$, Nur Fadilah Ab Aziz \\ Nur Azzammudin Rahmat ${ }^{5}$ \\ ${ }^{1,2,3}$ Faculty of Electrical Engineering, Universiti Teknologi MARA, Malaysia \\ ${ }^{4,5}$ Institute of Power Engineering, Electrical Power Department, College of Engineering, \\ Universiti Tenaga Nasional, Malaysia
}

\begin{tabular}{l} 
Article Info \\
\hline Article history: \\
Received Jan 3, 2019 \\
Revised Apr 6, 2019 \\
Accepted May 2, 2019 \\
\hline
\end{tabular}

Keywords:

Graphical user interface

Long transmission line

Medium transmission line

Short transmission line

Transmission line model

\begin{abstract}
Transmission line is one of the important elements in the process of power transfer from the source of generation to the consumer. In order to analyze the performance of a transmission line, it has to be represented by an equivalent model with suitable circuit parameters at a per phase basis. The line models are used to measure voltages, currents and the amount of power flow depending on the line length. Transmission line performance is determined by the voltage regulation and its efficiency under their normal operating conditions. In this study, a systematic approach was developed in order to assist the power system utility in planning the transmission line that needed to be installed in the system. With the aid of Graphical User Interface (GUI), the performance of transmission line can be determined and monitored due to the change of line parameters. The results obtained could assist the lecturers in delivering the concept of engineering in a more systematic approach. On top of that, it could also assist the power system utility in planning the transmission line that needed to be installed in the system.
\end{abstract}

Copyright (c) 2019 Institute of Advanced Engineering and Science. All rights reserved.

\section{Corresponding Author:}

Nur Ashida Salim,

Faculty of Electrical Engineering, Universiti Teknologi MARA,

40450 Shah Alam, Selangor, Malaysia.

Email: nurashida606@salam.uitm.edu.my

\section{INTRODUCTION}

In the 21st century, electrical grid system has been rapidly industrialize and becoming a critical part of the country's economic infrastructure. The rise of electrical production from renewable energy and the growing demand for electrical energy increase the importance of power transmission [1-3]. Transmission networks are designed by engineers to be efficient and feasible without neglecting on its safety, redundancy and also economic factor [4-6]. Transmission lines can be categorized into three categories which are short, medium and long transmission line. The classification of these lines are based on the distance from the sending end to the receiving end of the lines. Short transmission line has a limited distance of $80 \mathrm{~km}$, medium transmission line is between $81 \mathrm{~km}$ to $240 \mathrm{~km}$ while long transmission line is more than $240 \mathrm{~km}$ long [7-8].

Voltage drops, line losses and the transmission lines efficiency are vital consideration that needed in every design and operation of transmission lines. The line constant such as resistance (R), inductance (L), capacitance $(\mathrm{C})$ of the transmission have big influence on line performance. For an example, voltage drop of the line can be varied with the change in value of those line constant. Similarly, power loss in a transmission lines occurred due to the existence of the resistance in the transmission lines conductor. The parameters of the transmission lines contribute greatly on the lines performance. Resistance, inductance, capacitance and 
shunt conductance are the common parameters that exist in a transmission line [9-10]. All of those parameters known as distributed parameters since they are uniformly distributed along the transmission lines. Transmission lines are exposed with natural event such as lightning, heavy wind, hot climate that will reduce its performance [6, 11-13]. The integrity of the transmission lines is of the utmost importance, the performance of the transmission lines is determine by the voltage regulation and efficiency under their normal operating condition [1-2, 14-15]. According to [9], short transmission line is represented by a simple RL series equivalent circuit. The estimation of line parameter is the first step preceding the development of line model. By developing a dynamic model of the transmission line, the expected voltages at any locations of the transmission line either on the sources side or load sides can be predicted. The resistance of the conductors is the primary sources of transmission line losses [16]. As the current attempt to overwhelm the ohmic resistance of the line, useless heat is formed in the result of power dissipation.According to [17], AC transmission networks and distribution lines are purely reactive network. The existence of reactive power in the transmission lines increases the transmission losses. When the transmission line suffered too much of reactive power, the changes in load and load power factor will alter the voltage profile along the line and resulting in a huge amplitude variation at the receiving end voltage. Besides that, the abundancy of reactive power in the line will make the transmission line to be under voltage and also causing degradation in the performance of the loads especially induction motors. In the opposite of that, over voltage causes on resultant harmonic generation and damages on equipment due to insulation breakdowns [18]. According to [19], voltage drops occurred when the capacitive line charging current exist across the line inductance. The efficiency of the transmission line is proportional to the value of the capacitance in the transmission line. Steps can be taken to diminish losses to lead into efficient and effective operation of a power system [20]. The losses that sustained inside the resistance materials can be reduce by reducing the amount of current flow through the line, reducing the size of resistance or impedance along the transmission line and minimizing the level of voltage.

The scope of this study is mainly focusing on the development of a monitoring tool by using Graphical User Interface (GUI) in order to determine the performance of the transmission line. The biggest advantage of a GUI is that it works without prior knowledge and it is the most important aspect for any application [21-23]. A well designed GUIs are intuitive to the point that user need no knowledge about the underlying machinery at all. On the other hand, the main objective of this study is to model a transmission line using appropriate equivalent model where the performance due to change in the line parameters can be calculated and monitored through a systematic tool which is GUI.

\section{RESEARCH METHOD}

The performance of a transmission line shall have the measurement of the sending end and receiving end parameters, power factor, as well as voltage regulation and efficiency of the line model in percentage during its steady state and transient conditions [19, 24-25]. Performance calculations are helpful in system planning. In this study, the equations used to measure voltage drop and efficiency of transmission line shall be explained in detail. The project starts with identifying appropriate line model to be use in modelling the transmission lines [26]. Each of the model will have its own parameters that affect the efficiency of the transmission line. The calculations for each line model are then made in order to determine the performance of the transmission line. Then, the calculations for the performance of the line is developed into GUI. The GUI will display the results and summarize the parameters in the summary output. The project is considered success if the GUI able to achieve all the objectives for this project. As mentioned in the previous section, transmission lines can be categorized into three categories. Each of the categories is explained in detail in the following part.

\subsection{Short Line Model}

Figure 1 shows the circuit representation of a short line model which having voltage level that is not over $69 \mathrm{kV}$ and the length of the lines are less than $80 \mathrm{~km}$ is considered as short line model. The line capacitance may be often ignored without much error as the small leakage current, resistance, inductance are lumped along the transmission line. 


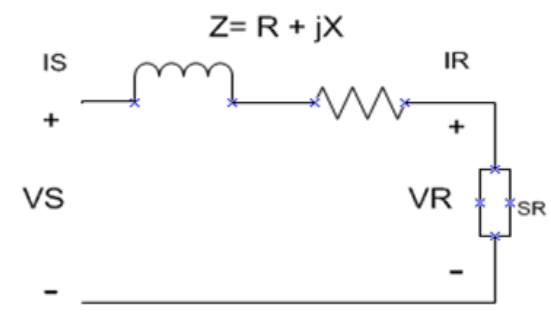

Figure 1. Short Line Model

The percentage change in voltage at the end of the receiving of the line can be defined as voltage regulation and can be calculated using (1) and the efficiency of the transmission line is given by (2).

$$
\begin{aligned}
& V_{R}=\frac{V_{R(\text { no load })}-V_{R(\text { full load })}}{V_{R(\text { full load })}} \\
& \eta=\frac{P_{R}}{P_{S}} \times 100 \%
\end{aligned}
$$

\subsection{Medium Line Model}

A medium line model is generally referred to a transmission lines that is in the range of $80 \mathrm{~km}$ to $250 \mathrm{~km}$. Half of the shunt capacitance is lumped at the end of the line as the line length is considerably lengthy. In this case, nominal $\pi$ model is used.

Figure 2 shows the circuit representation of the nominal $\pi$ model of medium line under a normal operating condition, the total shunt admittance of the line is given by (3).

$$
Y=(\overline{\mathrm{g}}+j \omega C) \ell \eta=\frac{P_{R}}{P_{S}} \times 100 \%
$$

Where $\overline{\mathrm{g}}$ is assumed to be zero as due to corona, the leakage current around the insulators is neglected. $C$ is the value of capacitance per kilometer from the line to neutral and $\ell$ is the length of the transmission line.

The percentage change in voltage at the end of the receiving of the line can be defined as voltage regulation and it can be obtained by using the same as (1). And finally, the efficiency of the transmission line can be calculated by using the same as (2).

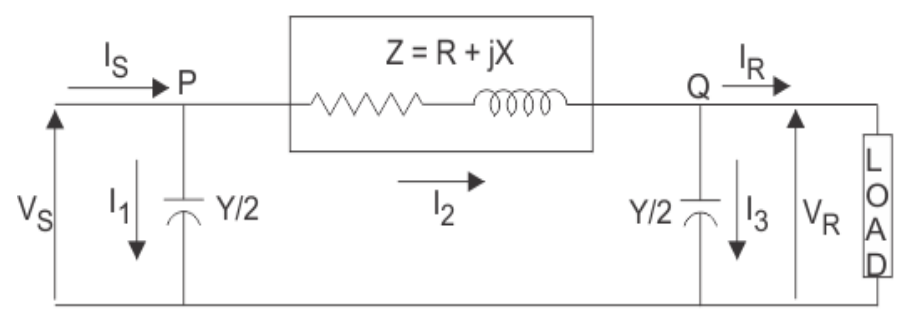

Figure 2. Nominal $\pi$ model of Medium Line

\subsection{Long Line Model}

To have more accurate modelling of a transmission line that is $250 \mathrm{~km}$ and longer, it cannot be assume that the line parameters are lumped since they are distributed along the lines. This long line model deals with complex calculations related to circuit parameters compared to short line or medium line model. The reason is that the long line model has higher effective circuit length compared to the former models. The long line model shown in Figure 3. 


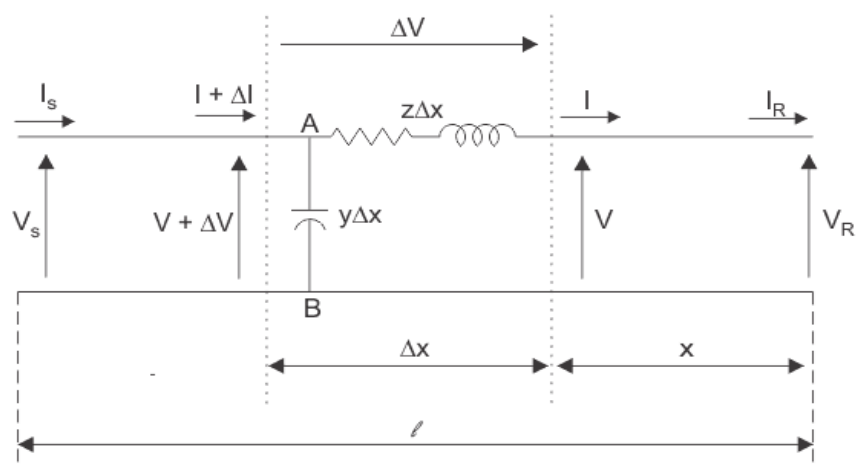

Figure 3. Long Line Model

By assuming this model is a lossless line, the line phase constant can be obtained by using (4).

$\beta=\omega \sqrt{L C}$

And the surge impedance is obtained by using (5).

$Z_{C}=\sqrt{\frac{L}{C}}$

As for the sending end voltage, it can be calculated using (6).

$V_{s}=\cos \beta \ell V_{R}+j Z_{C} \sin \beta \ell I_{R}$

The sending end current is obtained by using (7).

$$
I_{S}=\mathrm{j} \frac{1}{z_{C}} \sin \beta \ell I_{R}+\cos \beta \ell I_{R}
$$

The percentage change in voltage at the end of the receiving of the line can be defined as voltage regulation is obtained by using (8).

$$
V R=\frac{\frac{V_{R}}{\cos \beta \ell}-V_{R(\text { full load })}}{V_{R(\text { full load })}}
$$

\subsection{Graphical User Interface Model}

Figure 4 shows the flowchart for the process of graphical user interface in this project. Firstly, at the main page of the interface, the user needs to choose which line models that needed to be selected to undergo the analysis. Next, the user is directed to the second page of the interface where the user is allowed to insert the input parameters in the input section. Then, once the solve button is clicked, the GUI will execute the calculations and display the results on the performance of the selected line model in the output section as well as in the summary output. If the user wanted to insert new values for the parameter, it can done by pressing the Clear button to erase previous value and new values can be inserted. On the other hand, if the user wanted to do analysis on other line model, it can be done with a simple click of a button in the options box.

\section{RESULTS AND ANALYSIS}

With the aid of GUI, the analysis of the transmission line can be displayed interactively as shown in Figure 5. The implementation of GUI in this research give greater accessibility to the user. As soon as the GUI is executed, the home page will appear as shown in Figure 5. User are given options to choose which transmission line models to perform the analysis. By clicking on the type of the line model button, the GUI will redirect user to the second page of the GUI that will enable the user to perform calculation on the line models that has been selected. Figure 6 shows that overall interface of the project. 


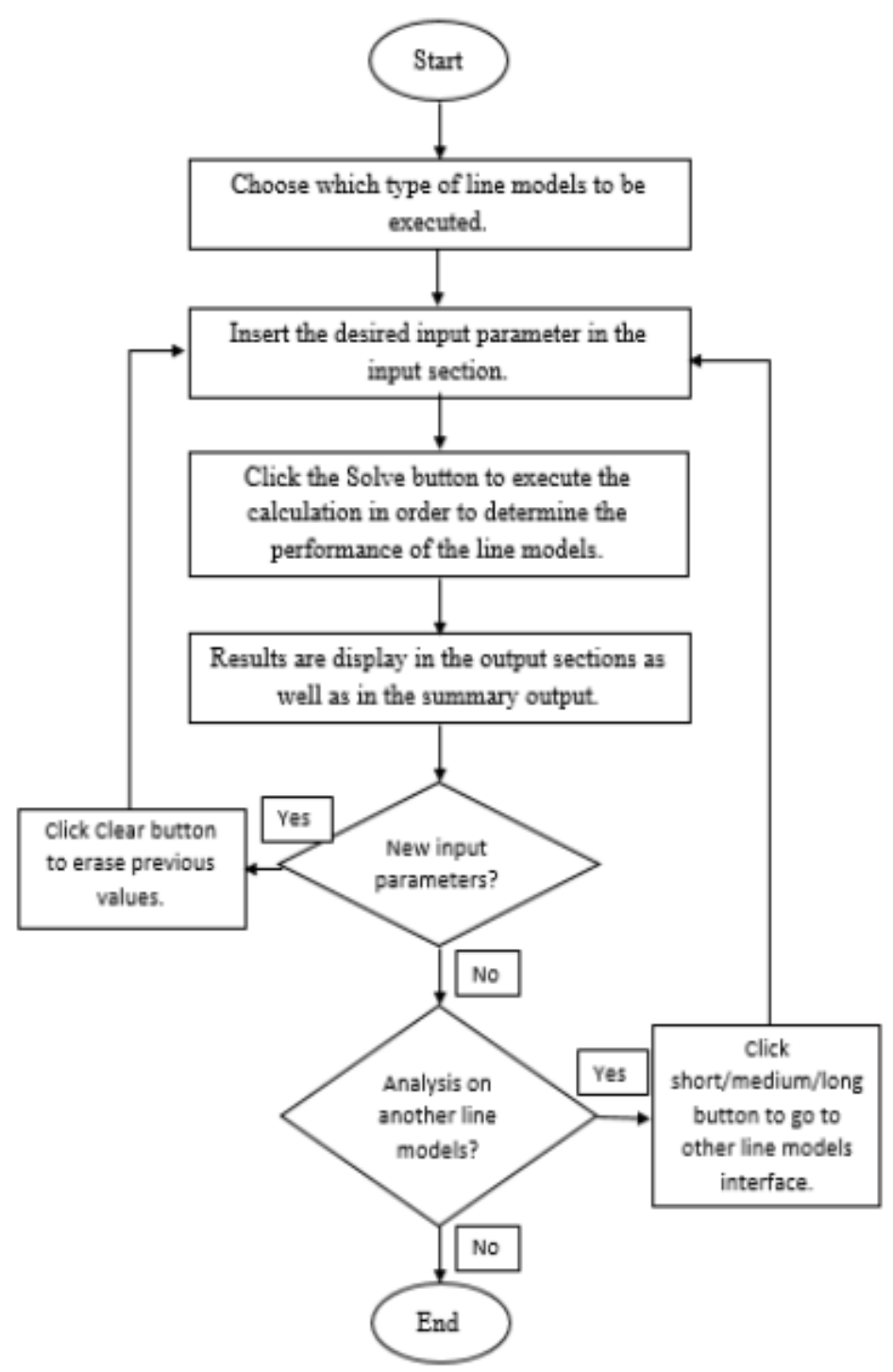

Figure 4. Flowchart of process for GUI

IMPLEMENTATION OF GUII IN DETERMINING THE PERFORMANCE OF TRANSMISSION LINES MODEL

\begin{tabular}{|c|c|c|}
\hline SHORT & MEDIUM & LONG \\
\hline & a & Anve 390 \\
\hline
\end{tabular}

Figure 5. Homepage of the GUI for the project 


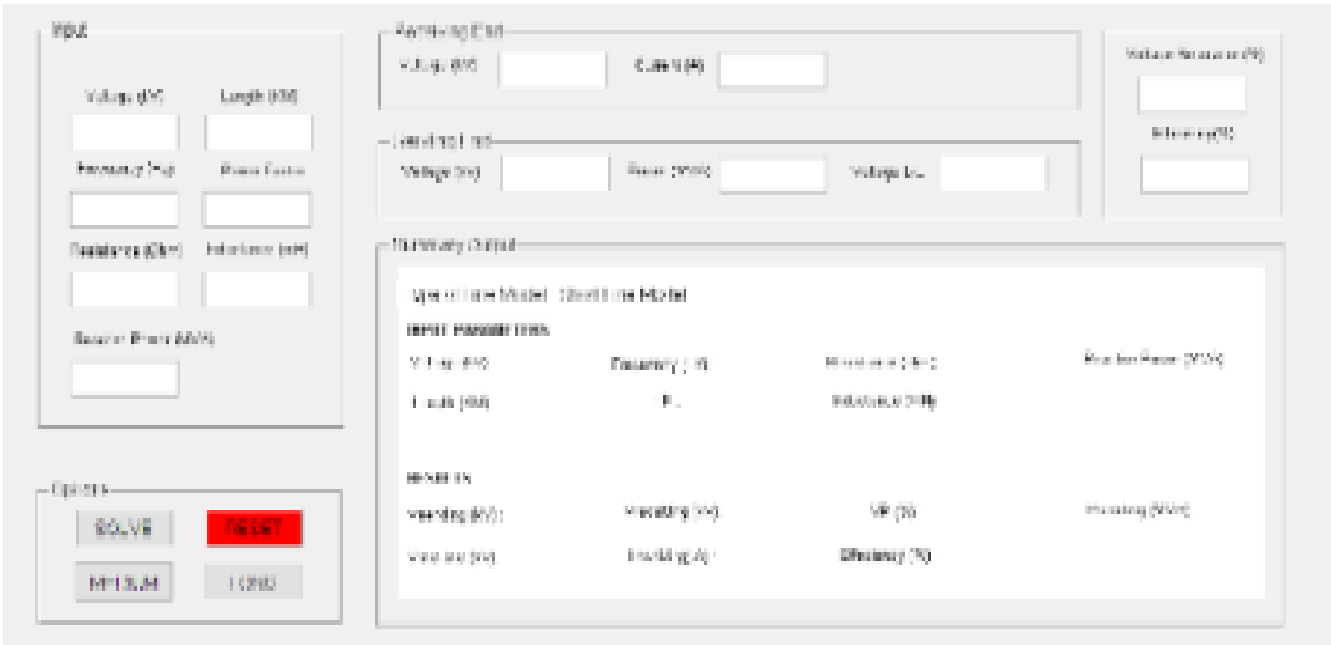

Figure 6. Overall look of the interface of the project

In the second page of the GUI, the interface consists of sections for inputs and outputs. This interface will display the information about the performance of the line models.

\subsection{Input Sections of the GUI}

For input sections, there will be seven parameters that user needs to key in the values in order to for the program to execute the simulation. The parameters are voltage, transmission line length, frequency, power factor, resistance, inductance and reactive power.

The parameters are inserted manually by clicking the desired parameter box and typing the value into it. The options box is located at the bottom of the input section. This options box offers 4 options to the user where each of the button has their own function.

a. Solve button.

Once this button is pressed, the GUI itself will perform calculations on the line model based on the formula that have been made and display the results in the output sections.

b. Reset button.

The button that coloured in red is the reset button where it will clear all the previous input and output that already have been display. Once it is executed, a new blank input and output are ready for new values to be inserted.

c. Short / Medium / Long button.

Each of the line model will have its correspondent button to redirect user to another interface for another different model of the line. For example, if the user is on the short model interface and wanted to go the long model interface, it can be done with a simple click of a button.

\subsection{Output Sections of the GUI}

Figure 7 shows the output section of the GUI where it displays results of the transmission line performance. The display will show the results of receiving end, sending end, voltage regulation and efficiency of the transmission line.

Figure 8 shows the summary output of the GUI. It summarizes all the input and output parameters that were used in determining the performance of the line model.

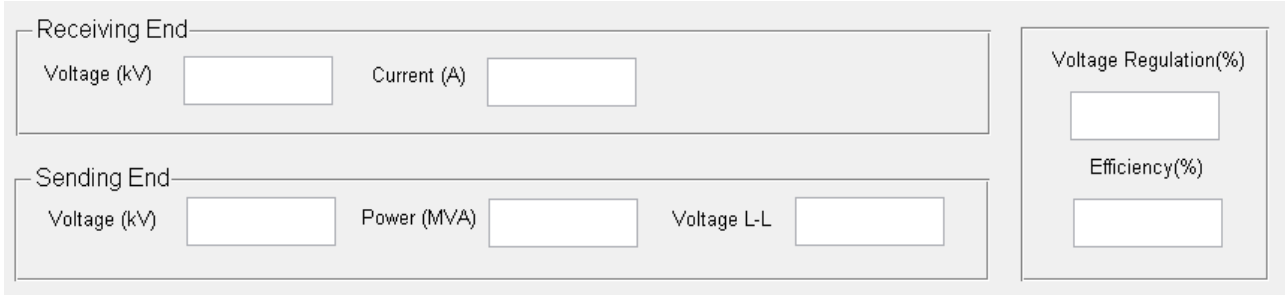

Figure 7. The interface for outputs 


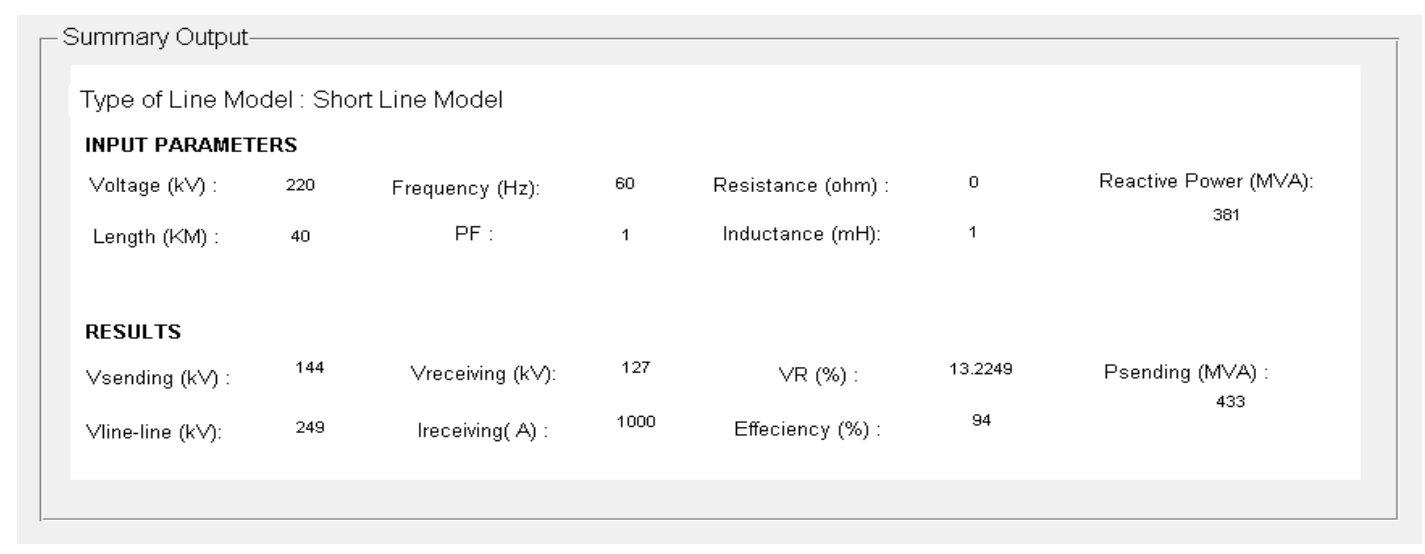

Figure 8. Summary output of the performance for the line model

\section{RESULTS OF THE TRANSMISSION LINE PERFORMANCE}

Table 1 shows the tabulation of data for the performance of the short transmission line. The performance of the transmission line is determined by its voltage regulation and efficiency.

When the resistance and the inductance of transmission line is increase, the performance of this short transmission line dropped significantly. As the current try to overcome the ohmic resistance of the line, losses occurred in the form of heat. Therefore, it can be seen clearly that for short transmission line, the efficiency of line is high when the resistance, inductance and capacitance in the line is low. However, the voltage regulation for short transmission line increases with respect to increase of the value of all the parameters.

Table 2 show the performance of the medium transmission line. The efficiency of the transmission line decreases gradually as the resistance and inductance of the transmission line increase. The value of the voltage regulation also dropped gradually in respect to the efficiency of the line. This also implies that for medium transmission line, the efficiency of the line is high with respect to low value of resistance and inductance. Similar to the short transmission line, the voltage regulation of medium transmission line increases with respect to the increase of the line parameters.

Table 1. Performance of Short Transmission Line

\begin{tabular}{cccc}
\hline $\begin{array}{c}\text { Resistance } \\
(\mathrm{Ohm})\end{array}$ & $\begin{array}{c}\text { Inductance } \\
(\mathrm{mH})\end{array}$ & $\begin{array}{c}\text { Voltage } \\
\text { Regulation }(\%)\end{array}$ & $\begin{array}{c}\text { Efficiency } \\
(\%)\end{array}$ \\
\hline 0.15 & 1.32 & 11.65 & 94.00 \\
0.20 & 1.33 & 12.93 & 93.00 \\
0.25 & 1.34 & 14.25 & 91.00 \\
0.30 & 1.35 & 15.56 & 89.00 \\
0.35 & 1.36 & 16.89 & 88.00 \\
0.40 & 1.37 & 18.21 & 86.00 \\
0.45 & 1.38 & 19.53 & 85.00 \\
0.50 & 1.39 & 20.85 & 84.00 \\
0.55 & 1.40 & 22.16 & 82.00 \\
0.60 & 1.41 & 23.48 & 81.00 \\
\hline
\end{tabular}

Table 2. Performance of Medium Transmission Line

\begin{tabular}{cccc}
\hline $\begin{array}{c}\text { Resistance } \\
(\mathrm{Ohm})\end{array}$ & $\begin{array}{c}\text { Inductance } \\
(\mathrm{mH})\end{array}$ & $\begin{array}{c}\text { Voltage } \\
\text { Regulation }(\%)\end{array}$ & $\begin{array}{c}\text { Efficiency } \\
(\%)\end{array}$ \\
\hline 0.15 & 0.80 & 9.15 & 94.69 \\
0.20 & 0.81 & 10.52 & 93.04 \\
0.25 & 0.82 & 11.90 & 91.45 \\
0.30 & 0.83 & 13.29 & 89.91 \\
0.35 & 0.84 & 14.69 & 88.42 \\
0.40 & 0.85 & 16.09 & 83.98 \\
0.45 & 0.86 & 17.49 & 85.59 \\
0.50 & 0.87 & 18.90 & 84.24 \\
0.55 & 0.88 & 20.32 & 82.94 \\
0.60 & 0.89 & 21.74 & 81.68 \\
\hline
\end{tabular}


Table 3 shows the performance of long transmission line when the value of capacitance is varied. As the capacitance increases, the magnitude of sending end voltage, VS decrease and making it lower than the magnitude of receiving end voltage, VR. Thus, the efficiency of the transmission line also drops gradually. Due to the line capacitance, voltage drop is produced by capacitive line charging current and the phenomenon is known as Ferranti effect. The results of efficiency and voltage regulation of long transmission line implies the same concept as for short and medium transmission lines.

Table 3. Performance of Long Transmission Line

\begin{tabular}{ccccc}
\hline $\begin{array}{c}\text { Capacitance } \\
(\mu \mathrm{F})\end{array}$ & $\begin{array}{c}\text { Sending end } \\
\text { voltage, } \mathrm{V}_{\mathrm{s}}\end{array}$ & $\begin{array}{c}\text { Receiving end } \\
\text { voltage, } \mathrm{V}_{\mathrm{R}}\end{array}$ & $\begin{array}{c}\text { Voltage } \\
\text { regulation }(\%)\end{array}$ & $\begin{array}{c}\text { Efficiency } \\
(\%)\end{array}$ \\
\hline 0.015 & 360.4 & 288.675 & 37.46 & 96.10 \\
0.02 & 350.7 & 288.675 & 38.34 & 90.10 \\
0.03 & 331.7 & 288.675 & 40.23 & 79.10 \\
0.04 & 313.1 & 288.675 & 42.36 & 68.95 \\
0.05 & 294.8 & 288.675 & 44.78 & 59.68 \\
0.06 & 276.9 & 288.675 & 47.54 & 51.25 \\
0.07 & 259.4 & 288.675 & 50.73 & 43.62 \\
0.08 & 242.2 & 288.675 & 54.44 & 36.75 \\
0.09 & 225.4 & 288.675 & 58.85 & 30.59 \\
0.10 & 208.9 & 288.675 & 64.13 & 25.11 \\
\hline
\end{tabular}

\section{CONCLUSION}

This paper has presented a systematic approach in determining the transmission line performance by using the Graphical user Interface. It is important to monitor the performance of a the transmission lines in order to ensure maximum power can be transferred to the consumer [27]. Therefore, the results obtained from this study could assist the power system utility in planning the transmission line that needed to be installed in the system depending the models of the transmission lines. The parameters of the transmission line contribute greatly to the performance of the line especially to its efficiency and voltage regulation. With appropriate model and accurate line parameter, the performance can be monitored. On top of that, the implementation of GUI makes the analysis of transmission lines more interactive. It is not only user-friendly, it is also owned low cognitive lode which means everything is laid in front of the user and the GUI itself takes care most of that freeing up the user mental processing power for the important stuff. As a conclusion, this GUI has been successfully implemented in order to monitor the performance of transmission line regardless it is a short, medium or long transmission line.

\section{ACKNOWLEDGEMENTS}

The authors would like to thank the Research Management Institute (RMI), Universiti Teknologi MARA, Malaysia and the Ministry of Higher Education (MOHE), Malaysia through research grant 600IRMI/DANA 5/3/BESTARI (121/2018) for the financial support of this research.

\section{REFERENCES}

[1] P. S. R. Murty, "Performance of Transmission Lines," in Electrical Power Systems, 2017.

[2] E. Bompard, R. Napoli, and F. Xue, "Analysis of structural vulnerabilities in power transmission grids," Int. J. Crit. Infrastruct. Prot., 2009.

[3] K. B. Adedeji, "Effect of HVTL Phase Transposition on Pipelines Induced Voltage," Indones. J. Electr. Eng. Informatics, 2016.

[4] H. H. Goh et al., "Transmission line fault detection: A review," Indonesian Journal of Electrical Engineering and Computer Science (IJEECS). 2017.

[5] S. H. Horowitz, "Transmission line protection," in Power System Stability and Control, Third Edition, 2017.

[6] N. Prasad Rao, G. M. Samuel Knight, S. J. Mohan, and N. Lakshmanan, "Studies on failure of transmission line towers in testing," Eng. Struct., 2012.

[7] J. M. Laheurte, "Transmission Line Model," in Compact Antennas for Wireless Communications and Terminals: Theory and Design, 2013.

[8] G. K. Reeves and H. B. Harrison, "Obtaining the Specific Contact Resistance from Transmission Line Model Measurements," IEEE Electron Device Lett., 1982.

[9] J. H. GRIDLEY, "Transmission-line Parameters," in Principles of Electrical Transmission Lines in Power and Communication, 2014.

[10] Y. Du and Y. Liao, "On-line estimation of transmission line parameters, temperature and sag using PMU measurements," Electr. Power Syst. Res., 2012. 
[11] R. Hui, I. Dobson, and B. A. Carreras, "Long-Term Effect of the n-1 Criterion on Cascading Line Outages in an Evolving Power Transmission Grid," IEEE Trans. Power Syst., vol. 23, no. 3, pp. 1217-1225, 2008.

[12] N. A. Salim, M. M. Othman, I. Musirin, M. S. Serwan, and S. Busan, "Risk assessment of dynamic system cascading collapse for determining the sensitive transmission lines and severity of total loading conditions," Reliab. Eng. Syst. Saf., vol. 157, pp. 113-128, 2017.

[13] S. Borenstein, J. Bushnell, and S. Stoft, "The Competitive Effects of Transmission Capacity in a Deregulated Electricity Industry," RAND J. Econ., 2006.

[14] M. J. Eppstein and P. D. H. Hines, "A 'Random Chemistry' Algorithm for Identifying Collections of Multiple Contingencies That Initiate Cascading Failure," IEEE Trans. Power Syst., vol. 27, no. 3, pp. 1698-1705, 2012.

[15] D. H. Slichter, O. Naaman, and I. Siddiqi, "Millikelvin thermal and electrical performance of lossy transmission line filters," Appl. Phys. Lett., 2009.

[16] M. Taleb, M. J. Ditto, and T. Bouthiba, "Performance of short transmission lines models," in 2006 IEEE GCC Conference, GCC 2006, 2006.

[17] M. Bočkarjova and G. Andersson, "Transmission line conductor temperature impact on state estimation accuracy," in 2007 IEEE Lausanne POWERTECH, Proceedings, 2007.

[18] M. C. Anumaka, "Analysis of Technical Losses in Electrical Power System ( Nigerian 330Kv Network As a Case Study )," Int. J. Res. Rev. Appl. Sci., 2012.

[19] S. H. M. Hashimoto, R. Romero, and J. R. S. Mantovani, "Efficient linear programming algorithm for the transmission network expansion planning problem," IEE Proc. - Gener. Transm. Distrib., 2003.

[20] C.-E. Sölver and S. de A. Morais, "Line-Charging Current Interruption by HV and EHV Circuit Breakers: Standard and Non-Standard Test Requirements as Determined by the Stresses Applied and by Breaker-Capability Considerations,” J. Syst. Simul., 2017.

[21] W. L. Martinez, “Graphical user interfaces,” Wiley Interdisciplinary Reviews: Computational Statistics. 2011.

[22] D. B. Nelson, M. H. Nehrir, and C. Wang, "Unit sizing and cost analysis of stand-alone hybrid wind/PV/fuel cell power generation systems," Renew. Energy, 2006.

[23] M. Dong, Y.-S. K. Choi, and L. Zhong, "Power modeling of graphical user interfaces on OLED displays," 2009.

[24] C. Bayliss and B. Hardy, Transmission and Distribution Electrical Engineering. 2012.

[25] N. B. Negra, J. Todorovic, and T. Ackermann, "Loss evaluation of HVAC and HVDC transmission solutions for large offshore wind farms,” Electr. Power Syst. Res., 2006.

[26] T. S. Kishore and S. K. Singal, "Optimal economic planning of power transmission lines: A review," Renewable and Sustainable Energy Reviews. 2014.

[27] IEEE Power Engineering Society, IEEE Std 1243-1997: IEEE Guide For Improving The lightning Performance of Transmission Lines. 1997. 